\title{
Computing the Minkowski Sum of Prisms
}

\author{
D. Pallaschke \\ Insitut für Statistik \\ und Mathem. Wirtschaftstheorie \\ Universität Karlsruhe \\ D-76128 Karlsruhe, Germany
}

\author{
J. Rosenmüller \\ Institut für \\ Mathematische Wirtschaftsforschung \\ Universität Bielefeld \\ D-33615 Bielefeld, Germany
}

Dedicated to the 65th birthday of Alexander Rubinov

\begin{abstract}
Within this paper we study the Minkowski sum of prisms ("Cephoids") in a finite dimensional vector space. For a vector $\boldsymbol{a} \in \mathbb{R}^{n}$ with positive components we write $\overline{\boldsymbol{a}}=\left(\frac{1}{\bar{a}_{1}}, \ldots, \frac{1}{\bar{a}_{n}}\right)$ and denote by $\boldsymbol{\Pi}=\boldsymbol{\Pi}^{\overline{\boldsymbol{a}}}=\{\boldsymbol{x} \in$ $\left.\mathbb{R}^{n} \mid\langle\overline{\boldsymbol{a}}, \boldsymbol{x}\rangle \leq 1, \quad \boldsymbol{x} \geq 0\right\}$ the associated prism. We provide a representation of a finite sum of prisms in terms of inequalities.
\end{abstract}

Keywords: Convex analysis, Minkowski sum, polytopes.

2000 Mathematical Subject Classification: 52A07, 26A27, 90C08

\section{Introduction}

For two arbitrary subsets $A, B \subset \mathbb{R}^{n}$ the Minkowski sum is defined by the formula $A+B=\{\boldsymbol{z}=\boldsymbol{a}+\boldsymbol{b} \mid \boldsymbol{a} \in A, \boldsymbol{b} \in B\}$ and for $\lambda \in \mathbb{R}$ and $A \subset \mathbb{R}^{n}$ the multiplication is defined by $\lambda A=\{\boldsymbol{x}=\lambda \boldsymbol{a} \mid \boldsymbol{a} \in A\}$. If $A$ and $B$ are convex sets then the sets $A+B$ and $\lambda A$ are also convex and if moreover $A$ and $B$ are polytopes, then $A+B$ and $\lambda A$ are also polytopes. Thus the Minkowski sum of finitely many polytopes is the convex hull of the sum of its extreme points.

In addition to this representation it is frequently helpful to have a dual representation of the Minkowski sum of finitely many polytopes in terms of inequalities. One point is that the Minkowski sums of prisms are the basic objects in cooperative game theory (see [5], [4]). Another point is that they constitute the feasible 
regions of generalized knapsack problems, that is, of linear optimization problems of the type:

under

$$
\max \langle\boldsymbol{c}, \boldsymbol{x}\rangle
$$

with

$$
\boldsymbol{x}=\boldsymbol{z}^{1}+\boldsymbol{z}^{2}+\ldots+\boldsymbol{z}^{k}
$$

$$
\begin{aligned}
& \left\langle\boldsymbol{a}^{i}, \boldsymbol{z}^{i}\right\rangle \leq \alpha_{i} \\
& \boldsymbol{z}^{i} \geq 0
\end{aligned}
$$

with $\boldsymbol{c}, \boldsymbol{a}^{i}, \boldsymbol{z}^{i} \in \mathbb{R}^{n}$ and $\alpha_{i} \in \mathbb{R}$.

Structural properties about the Minkowski sum of prisms, such as the number of faces and symmetries for the generic case have been studied in [3]. We have called a finite sum of prisms a "cephoid".

Contrary to these investigations, we do not focus on the generic or "nondegenerate" case in the present paper. As a consequence, the results are weaker but more general. In particular, we provide a version of the coincidence theorem which yields a necessary and sufficient condition for maximal faces - but lacks the specific enumeration of the coordinates as specified in [3]

This version turns out to be much more suitable for computational purposes. When computing the maximal faces of cephoids, we do not want to test for nondegeneracy in advance. Hence, there is a particular emphasis on computational aspects in the present paper.

Let us denote by $\mathbf{0} \in \mathbb{R}^{n}$ the origin of and by $\boldsymbol{e}^{i}$ the $i$-th unit vector of $\mathbb{R}^{n}$ for $i \in\{1, \ldots, n\}$. For a vector $\boldsymbol{a}=\left(a_{1}, \ldots, a_{n}\right)>0 \in \mathbb{R}^{n}$, let

$$
\boldsymbol{\Pi}^{\boldsymbol{a}}=\operatorname{conv}\left(\left\{\mathbf{0}, \boldsymbol{a}^{1}, \ldots, \boldsymbol{a}^{n}\right\}\right)
$$

with $\boldsymbol{a}^{i}=a_{i} \boldsymbol{e}^{i}, \quad i \in\{1, \ldots, n\}$. We call $\boldsymbol{\Pi}^{\boldsymbol{a}}$ a prism associated to the vector $\boldsymbol{a}=\left(a_{1}, \ldots, a_{n}\right)>\mathbf{0} \in \mathbb{R}^{n}$.

Observe that $\boldsymbol{\Pi}^{\boldsymbol{a}}=\left\{\boldsymbol{x} \in \mathbb{R}^{n} \mid\langle\overline{\boldsymbol{a}}, \boldsymbol{x}\rangle \leq \alpha\right.$ and $\left.\boldsymbol{x} \geq \mathbf{0}\right\}$, where $\overline{\boldsymbol{a}}=\left(\frac{\alpha}{a_{1}}, \ldots, \frac{\alpha}{a_{n}}\right) \in$ $\mathbb{R}^{n}$ is called the outer normal (for level $\alpha>0$ ) of $\boldsymbol{\Pi}^{a}$. In this paper we will always choose $\alpha=1$.

We call

$$
\boldsymbol{\Delta}^{\boldsymbol{a}}=\operatorname{conv}\left(\left\{\boldsymbol{a}^{1}, \ldots, \boldsymbol{a}^{n}\right\}\right)
$$

the Pareto face of $\Pi^{a}$.

Whenever we have finitely many vectors, say $\boldsymbol{a}^{(1)}, \ldots, \boldsymbol{a}^{(K)} \in \mathbb{R}^{n}$ with $\boldsymbol{a}^{(k)}=$ $\left(a_{1}^{(k)}, \ldots, a_{n}^{(k)}\right)>\mathbf{0} \in \mathbb{R}^{n}$, we will write for the corresponding prisms

$$
\boldsymbol{\Pi}^{(k)}=\boldsymbol{\Pi}^{\boldsymbol{a}^{(k)}}=\operatorname{conv}\left(\left\{\mathbf{0}, \boldsymbol{a}^{(k), 1}, \ldots, \boldsymbol{a}^{(k), n}\right\}\right)
$$


with $\boldsymbol{a}^{(k), i}=a_{i}^{(k)} \boldsymbol{e}^{i}, \quad i \in\{1, \ldots, n\}, k \in\{1, \ldots, K\}$

\section{The Decomposition Principle}

Henceforth we write $\boldsymbol{I}=\{1, \ldots, n\}$. For the convex hull of the extreme points $\boldsymbol{a}^{i_{1}}, \ldots, \boldsymbol{a}^{i_{r}}$ of the prism $\boldsymbol{\Pi}^{a}$ we will use the abbreviation

$$
\operatorname{conv}\left(\left\{\boldsymbol{a}^{i_{1}}, \ldots, \boldsymbol{a}^{i_{r}}\right\}\right)=\left[\boldsymbol{a}^{i_{1}}, \ldots, \boldsymbol{a}^{i_{r}}\right]=\boldsymbol{\Delta}_{\boldsymbol{J}}^{(\boldsymbol{a})},
$$

with $\boldsymbol{J}=\left\{i_{1}, \ldots, i_{r}\right\}$. For a collection of prisms $\boldsymbol{\Pi}^{\boldsymbol{a}^{(k)}}, k=1, \ldots, K$, we will write

$$
\boldsymbol{\Delta}_{\boldsymbol{J}^{(k)}}^{(k)}=\boldsymbol{\Delta}_{\boldsymbol{J}^{(k)}}^{(\boldsymbol{a})^{(k)}}
$$

for the convex hull of the extreme points

$$
\left\{\boldsymbol{a}^{(k), l}\right\}_{l \in \boldsymbol{J}^{(k)}} \subset \boldsymbol{\Pi}^{a^{(k)}}
$$

whenever $\boldsymbol{J}^{(k)} \subseteq \boldsymbol{I}$ is subset of coordinates.

\section{Lemma 2.1.}

For a vector $\boldsymbol{a}=\left(a_{1}, \ldots, a_{n}\right)>\mathbf{0} \in \mathbb{R}^{n}$ let $\boldsymbol{\Pi}^{\boldsymbol{a}}$ be the associated prism. Then, for every extreme set $S \subset \boldsymbol{\Delta}^{a}$ there exist $\boldsymbol{J} \subseteq \boldsymbol{I}$ such that

$$
S=\operatorname{conv}\left(\left\{\boldsymbol{a}^{i}\right\}\right)_{i \in \boldsymbol{J}}=\boldsymbol{\Delta}_{\boldsymbol{J}}^{(\boldsymbol{a})} .
$$

Proof: Let $S \subset \boldsymbol{\Pi}^{a}$ be an extreme set of the Pareto-face $\boldsymbol{\Delta}^{a}$ of $\boldsymbol{\Pi}^{a}$. Then by definition of an extreme set we have that for every two points $\boldsymbol{u}, \boldsymbol{v} \in \boldsymbol{\Pi}^{\boldsymbol{a}}$ whose line-segment $[\boldsymbol{u}, \boldsymbol{v}]$ intersects $S$, i.e. $[\boldsymbol{u}, \boldsymbol{v}] \cap S \neq \emptyset$, it follows that $\boldsymbol{u}, \boldsymbol{v} \in S$. If we perform this procedure with the extreme points of $\Pi^{a}$ we get a collection of extreme points $\left\{\boldsymbol{a}^{i}\right\}_{i \in \boldsymbol{J}}$ of $\boldsymbol{\Pi}^{\boldsymbol{a}}$ which are elements of $S$. Hence it follows that

$$
S=\operatorname{conv}\left(\left\{\boldsymbol{a}^{i}\right\}\right)_{i \in \boldsymbol{J}}=\boldsymbol{\Delta}_{\boldsymbol{J}}^{(\boldsymbol{a})}
$$

holds true which proves the assertion.

q.e.d.

The key for all investigations of the structure of the Minkowski sum of prisms or cephoids is the theorem on the addition of faces (see [6] and [7]), which we will state here in the following way:

Lemma 2.2 (The Decomposition of Faces). Let $\boldsymbol{a}^{(1)}, \ldots, \boldsymbol{a}^{(K)} \in \mathbb{R}^{n}$ be a family of positive vectors and let

$$
\boldsymbol{\Pi}=\sum_{k=1}^{K} \boldsymbol{\Pi}^{(k)}
$$

with $\boldsymbol{\Pi}^{(k)}=\Pi^{a^{(k)}}$ for $k \in\{1, \ldots, K\}$ be the generated cephoid. Let $F \subset \boldsymbol{\Pi}$ be a maximal face of $\boldsymbol{\Pi}$, i.e. $\operatorname{dim} F=(n-1)$ and let $\mathfrak{n}_{F} \in \mathbb{R}^{n}$ be an outer normal of the face $F$, i.e. 


$$
F=\boldsymbol{\Pi} \cap\left\{\boldsymbol{x} \in \boldsymbol{\Pi} \mid\left\langle\mathfrak{n}_{F}, \boldsymbol{x}\right\rangle=d^{*}\right\}, \quad j=1, \ldots, k,
$$

where

$$
d^{*}=\max _{\boldsymbol{x} \in \boldsymbol{\Pi}}\left\langle\mathfrak{n}_{F}, x\right\rangle>0 .
$$

Then there exists $\boldsymbol{K}_{0} \subseteq\{1, \ldots, K\}$ and index sets $\boldsymbol{J}^{(k)} \subseteq \boldsymbol{I}\left(k \in \boldsymbol{K}_{0}\right)$ such that

$$
F=\sum_{k \in \boldsymbol{K}_{0}} \boldsymbol{\Delta}_{\boldsymbol{J}^{(k)}}^{(k)}+\boldsymbol{p}
$$

where $\boldsymbol{p}$ is a sum of extreme points of the remaining prisms $\left\{\boldsymbol{\Pi}^{(k)}\right\}_{k \notin \boldsymbol{K}_{0}}$.

Proof: By [1] Theorem 1.5 there exist prisms $\boldsymbol{\Pi}^{\left(i_{1}\right)}, \ldots, \boldsymbol{\Pi}^{\left(i_{r}\right)}$ such that

$$
F=S_{i_{1}}+\ldots+S_{i_{r}}+\boldsymbol{p}
$$

with

$$
S_{i_{j}}=\left\{\boldsymbol{x} \in \mathbf{\Pi}^{\left(i_{j}\right)} \mid\left\langle\mathfrak{n}_{F}, x\right\rangle=d^{i_{j}}\right\}, \quad j=1, \ldots, r,
$$

where $d^{i_{j}}=\max _{\boldsymbol{x} \in \boldsymbol{\Pi}^{i_{j}}}\left\langle\mathfrak{n}_{F}, x\right\rangle>0$ and $\boldsymbol{p}$ is a sum of extreme points of the remaining prisms.

Put $\boldsymbol{K}_{0}=\left\{i_{1}, \ldots, i_{r}\right\}$. Since every $S_{k}$ is an extreme subset of the Pareto face of $\boldsymbol{\Pi}^{(k)}$, it can be written as

$$
S_{k}=\Delta_{\boldsymbol{J}^{(k)}}^{(k)}
$$

with a suitable subset $\boldsymbol{J}^{(k)} \subseteq \boldsymbol{I}$.

q.e.d.

Hence every maximal face contains a translate of the Minkowski sum of non-zero dimensional extreme sets of prisms. Observe that the outer normals of the maximal face and the Minkowski sum of non-zero dimensional extreme sets of prisms are equal up to a nonnegative multiple.

Henceforth we use $\boldsymbol{K}=\{1, \ldots, K\}$ for the index set of a collection of positive vectors. In view of the above presentation we assign to every maximal face

$$
F \subset \boldsymbol{\Pi}=\sum_{k=1}^{K} \boldsymbol{\Pi}^{(k)}=\sum_{k \in \boldsymbol{K}} \boldsymbol{\Pi}^{(k)}
$$

the following collection of pairs of index sets

$$
\mathcal{J}_{F}=\left(\boldsymbol{K}_{0},\left[\boldsymbol{J}^{(k)}\right]_{k \in \boldsymbol{K}_{0}}\right)
$$

Here $\boldsymbol{K}_{0} \subset \boldsymbol{K}$ is the set of indices of those prisms $\boldsymbol{\Pi}^{(k)}$, the face of which is not zero-dimensional with respect to $\mathfrak{n}_{F}$, i.e., that satisfy

$$
\operatorname{dim}\left(S_{k}\right)=\operatorname{dim}\left(\left\{\boldsymbol{x} \in \boldsymbol{\Pi}^{(k)} \mid\left\langle\mathfrak{n}_{F}, \boldsymbol{x}\right\rangle=d^{k}\right\}\right) \geq 1
$$

with $d^{k}=\max _{\boldsymbol{x} \in \boldsymbol{\Pi}^{k}}\left\langle\mathfrak{n}_{F}, \boldsymbol{x}\right\rangle>0$. Also, $\boldsymbol{J}^{(k)}$ contains the indices of the extreme points of this face in $\boldsymbol{\Pi}^{(k)}$. We call set $\mathcal{J}_{F}$ the canonical reference system of $F$. 


\section{Proposition 2.3.}

Let $\boldsymbol{a}^{(1)}, \ldots, \boldsymbol{a}^{(K)} \in \mathbb{R}^{n}$ be a family of positive vectors and let

$$
\boldsymbol{\Pi}=\sum_{k=1}^{K} \boldsymbol{\Pi}^{(k)}
$$

with $\Pi^{(k)}=\Pi^{a^{(k)}}$ for $k \in \boldsymbol{K}$. Let $F \subset \boldsymbol{\Pi}$ be a maximal face and $\mathfrak{n}_{F} \in \mathbb{R}^{n}$ be an outer normal of the face $F$.

Then $\mathfrak{n}_{F}$ is a strictly positive vector.

Proof: Let us assume that $F \subset \boldsymbol{\Pi}$ is a maximal face of $\boldsymbol{\Pi}$ and that $\mathfrak{n}_{F}=\left(\alpha_{1} \ldots \alpha_{n}\right) \in$ $\mathbb{R}^{n}$ is an outer normal with level 1 of $F$. Then

$$
F=\left\{\boldsymbol{x} \in \boldsymbol{\Pi} \mid\left\langle\mathfrak{n}_{F}, \boldsymbol{x}\right\rangle=1\right\} .
$$

By the decomposition principle there exist prisms $\boldsymbol{\Pi}^{(k)}\left(k \in \boldsymbol{K}_{0}\right)$ such that

$$
F=\sum_{k \in \boldsymbol{K}_{0}} \boldsymbol{\Delta}_{\boldsymbol{J}^{(k)}}^{(k)}+\boldsymbol{p}
$$

satisfying

$$
\boldsymbol{\Delta}_{\boldsymbol{J}^{(k)}}^{(k)}=\left\{\boldsymbol{x} \in \boldsymbol{\Pi}^{(k)} \mid\left\langle\mathfrak{n}_{F}, \boldsymbol{x}\right\rangle=d^{i_{j}}\right\}, \quad k \in \boldsymbol{K}_{0}
$$

where $d^{k}=\max _{\boldsymbol{x} \in \boldsymbol{\Pi}^{k}}\left\langle\mathfrak{n}_{F}, \boldsymbol{x}\right\rangle>0$ and $\boldsymbol{p}$ is a sum of extreme points of the remaining prisms. Moreover

$$
\left\langle\mathfrak{n}_{F}, \boldsymbol{a}^{(k) i}\right\rangle=d^{k}\left(i \in \boldsymbol{J}^{(k)}\right)
$$

Since

$$
\left\langle\mathfrak{n}_{F}, \boldsymbol{a}^{(k), i}\right\rangle=\alpha_{i} a_{i}^{(k)}
$$

it follows that $\alpha_{i}>0$ and from the assumption that $F$ is a maximal face, i.e. $\operatorname{dim} F=n-1$, it follows that all components of $\mathfrak{n}_{F}$ are greater than zero. q.e.d.

\section{Theorem 2.4.}

Let $\boldsymbol{a}^{(1)}, \ldots, \boldsymbol{a}^{(K)} \in \mathbb{R}^{n}$ be a family of positive vectors and let

$$
\boldsymbol{\Pi}=\sum_{k=1}^{K} \boldsymbol{\Pi}^{(k)}
$$

with $\boldsymbol{\Pi}^{(k)}=\boldsymbol{\Pi}^{a^{(k)}}$ for $k \in \boldsymbol{K}$. Let $F \subset \boldsymbol{\Pi}$ be a maximal face of $\boldsymbol{\Pi}$ with outer normal $\mathfrak{n}_{F} \in \mathbb{R}^{n}$ and let with suitable $\boldsymbol{K}_{0} \subseteq \boldsymbol{K}$

$$
F=\sum_{k \in \boldsymbol{K}_{0}} \boldsymbol{\Delta}_{\boldsymbol{J}^{(k)}}^{(k)}+\boldsymbol{p}
$$

with

$$
\boldsymbol{\Delta}_{\boldsymbol{J}^{(k)}}^{(k)}=\left\{\boldsymbol{x} \in \boldsymbol{\Pi}^{(k)} \mid\left\langle\mathfrak{n}_{F}, \boldsymbol{x}\right\rangle=d^{k}\right\}, \quad k \in \boldsymbol{K}_{0}
$$

where $d^{k}=\max _{\boldsymbol{x} \in \boldsymbol{\Pi}^{k}}\left\langle\mathfrak{n}_{F}, \boldsymbol{x}\right\rangle>0$ and $\boldsymbol{p}$ is a sum of extreme points of the remaining prisms. Then, for any decomposition of $\boldsymbol{I}=\{1, \ldots, n\}$ into two nonempty disjoint subsets $\boldsymbol{I}_{0}$ and $\boldsymbol{I}_{1}$ there exists $\kappa \in \boldsymbol{K}_{0}$ such that $\boldsymbol{J}^{(\kappa)}$ has a nonempty intersection with both sets $\boldsymbol{I}_{0}$ and $\boldsymbol{I}_{1}$. 
Proof: Let us assume that the assertion is not true. Then there exists a decomposition of $\{1, \ldots, n\}$ into two nonempty disjoint subsets $\boldsymbol{I}_{0}$ and $\boldsymbol{I}_{1}$ such that every set $\boldsymbol{J}^{(k)}$ from the canonical reference system of $F$ belongs either to $\boldsymbol{I}_{0}$ or to $\boldsymbol{I}_{1}$.

Now, to every $\kappa \in \boldsymbol{K}_{0}$ we assign a fixed index $i_{k} \in \boldsymbol{J}^{(k)}$ and a row-vector

$$
\widehat{\boldsymbol{a}}^{(k)}=\sum_{i \in \boldsymbol{J}^{(k)}} \boldsymbol{a}^{(k) i}-\boldsymbol{a}^{(k) i_{k}}
$$

Since

$$
\left.\left\langle\mathfrak{n}_{F}, \boldsymbol{a}^{(k) i}\right)\right\rangle=\left\langle\mathfrak{n}_{F}, \boldsymbol{a}^{(k) i_{k}}\right\rangle \quad\left(i \in \boldsymbol{J}^{(k)},\right.
$$

it follows that the above row-vector is orthogonal to the outer normal vector $\mathfrak{n}_{F}$.

Now we build a matrix that involves all these row-vectors. Necessarily, after a suitable permutation of rows and columns, this matrix has the following form:

$$
M=\left(\begin{array}{c|cc}
\cdots \boldsymbol{I}_{0} \cdots & \cdots & \boldsymbol{I}_{1} \cdots \\
A & & \mathbf{0} \\
\hline \mathbf{0} & & B
\end{array}\right) .
$$

Since the outer normal vector $\mathfrak{n}_{F}$ is uniquely determined up to a positive multiple and belongs to the kernel of the above matrix $M$, we deduce, that the rank of $M$ is $(n-1)$.

If both sub-matrices $A$ and $B$ do not have full rank, then $M$ has at most rank $(n-2)$ which is not possible, so we can assume, that $B$ has full rank and $A$ not. Hence the equation $M \mathfrak{n}_{F}=0$ has a solution, where all components of $\mathfrak{n}_{F}$ with indices in $\boldsymbol{I}_{1}$ vanish and this is a contradiction to Proposition 2.3.

q.e.d.

\section{The Coincidence Theorem}

Given two convex sets $A, B \subset \mathbb{R}^{n}$ we denote by

$$
A \vee B=\operatorname{conv}(A \cup B)
$$

the convex hull of their union. Also we write

$$
\boldsymbol{\Pi}_{\boldsymbol{J}}^{\boldsymbol{a}}=\boldsymbol{\Delta}_{\boldsymbol{J}}^{\boldsymbol{a}} \vee\{\mathbf{0}\}
$$

for the prism generated by an extreme set of $\boldsymbol{\Delta}^{a}$. The notation $\boldsymbol{\Pi}_{\boldsymbol{J}^{(k)}}^{(k)}$ then refers to the vector $\boldsymbol{a}^{(k)}$ of a family. Then we have: 


\section{Theorem 3.1.}

Let $\boldsymbol{a}^{(1)}, \ldots, \boldsymbol{a}^{(K)} \in \mathbb{R}^{n}$ be a family of positive vectors and let

$$
\boldsymbol{\Pi}=\sum_{k=1}^{K} \boldsymbol{\Pi}^{(k)}
$$

with $\boldsymbol{\Pi}^{(k)}=\boldsymbol{\Pi}^{a^{(k)}}$ for $k \in\{1, \ldots, m\}$. Let $F \subset \boldsymbol{\Pi}$ be a maximal face of $\boldsymbol{\Pi}$.

Then a vector $\mathfrak{n}_{F} \in \mathbb{R}^{n}$ is an outer normal (to the level 1) of the maximal face $F \subset \boldsymbol{\Pi}$ if and only if there exist $\boldsymbol{K}_{0} \subset \boldsymbol{K}$ and faces

$$
\boldsymbol{\Delta}_{\boldsymbol{J}^{(k)}}^{(k)} \subset \boldsymbol{\Pi}^{(k)}, \quad k \in \boldsymbol{K}_{0}
$$

as well as positive numbers $c_{k} \in \mathbb{R}$ which satisfy the following conditions:

1. For every $k \in \boldsymbol{K}_{0}$ the linear function $x \mapsto\left\langle n_{F} \boldsymbol{x}\right\rangle$ is constant equal to 1 on the face $c_{k} \boldsymbol{\Delta}_{\boldsymbol{J}^{(k)}}^{(k)}$.

2. if, for some $k, l \in \boldsymbol{K}_{0}$, two different faces $c_{k} \boldsymbol{\Delta}_{\boldsymbol{J}^{(k)}}^{(k)}$ and $c_{l} \boldsymbol{\Delta}_{\boldsymbol{J}^{(l)}}^{(l)}$ have extreme points which lie on the same coordinate axis, then these extreme points coincide.

3. The prism

$$
\widehat{\boldsymbol{\Pi}}=\bigvee_{k \in \boldsymbol{K}_{0}} c_{k} \boldsymbol{\Pi}_{\boldsymbol{J}^{(k)}}^{(k)}
$$

is n-dimensional and its outer normal (to the level 1 ) is the outer normal $\mathfrak{n}_{F}$ of $F$.

Proof: Let us assume that $F \subset \boldsymbol{\Pi}$ is a maximal face of $\boldsymbol{\Pi}$ and that $\mathfrak{n}_{F} \in \mathbb{R}^{n}$ is an outer normal of $F$. Then

$$
F=\left\{\boldsymbol{x} \in \boldsymbol{\Pi} \mid\left\langle\mathfrak{n}_{F} \boldsymbol{x}\right\rangle=1\right\} .
$$

By the decomposition principle there exist prisms $\boldsymbol{\Pi}^{(k)} k \in \boldsymbol{K}_{0}$ such that

$$
F=\sum_{k \in \boldsymbol{K}_{0}} \boldsymbol{\Delta}_{\boldsymbol{J}^{(k)}}^{(k)}+\boldsymbol{p}
$$

where $\boldsymbol{p}$ is a sum of extreme points of the remaining prisms with index $k \notin \boldsymbol{K}_{0}$. Again we know that with

$$
\boldsymbol{\Delta}_{\boldsymbol{J}^{(k)}}^{(k)}=\left\{\boldsymbol{x} \in \boldsymbol{\Pi}^{(k)} \mid\left\langle\mathfrak{n}_{F}, \boldsymbol{x}\right\rangle=d^{k}\right\}, \quad k \in \boldsymbol{K}_{0},
$$

with $d^{k}=\max _{\boldsymbol{x} \in \boldsymbol{\Pi}^{(k)}}\left\langle\mathfrak{n}_{F}, \boldsymbol{x}\right\rangle>0$.

Now observe that the faces

$$
\boldsymbol{\Delta}_{\boldsymbol{J}^{(k)}}^{(k)} \quad\left(k \in \boldsymbol{K}_{0}\right)
$$

are lying in parallel hyperplanes, namely

$$
H^{k}=\left\{z \in \mathbb{R}^{n} \mid\left\langle\mathfrak{n}_{F}, \boldsymbol{x}\right\rangle=d^{k}\right\}, \quad k \in \boldsymbol{K}_{0},
$$


with $d^{k}>0 \quad\left(k \in \boldsymbol{K}_{0}\right)$. Now choose

$$
c_{k}=\frac{1}{d^{k}}, \quad k \in \boldsymbol{K}_{0} .
$$

Then the first condition is satisfied.

Now assume that two faces $c_{k} \boldsymbol{\Delta}_{\boldsymbol{J}^{(k)}}^{(k)}$ and $c_{l} \boldsymbol{\Delta}_{\boldsymbol{J}^{(l)}}^{(l)}$ have extreme points which lie on the $j$-th coordinate axis, i.e

$$
c_{k} \boldsymbol{a}_{j}^{(k)} \boldsymbol{e}^{j} \in c_{k} \boldsymbol{\Delta}_{\boldsymbol{J}^{(k)}}^{(k)}
$$

and

$$
c_{l} \boldsymbol{a}_{j}^{(l)} \boldsymbol{e}^{j} \in c_{l} \boldsymbol{\Delta}_{\boldsymbol{J}^{(l)}}^{(l)}
$$

Then

$$
\left\langle\mathfrak{n}_{F}, \boldsymbol{a}_{j}^{(k)} \boldsymbol{e}^{j}\right\rangle=d^{k}
$$

and

$$
\left\langle\mathfrak{n}_{F}, \boldsymbol{a}_{j}^{(l)} \boldsymbol{e}^{j}\right\rangle=d^{l},
$$

hence

$$
\frac{d^{k}}{\boldsymbol{a}_{j}^{(k)}}=\frac{d^{l}}{\boldsymbol{a}_{j}^{(l)}}
$$

which means that

$$
c_{k} \boldsymbol{a}_{j}^{(k)}=c_{l} \boldsymbol{a}_{j}^{(l)} .
$$

Hence, the two extreme points coincide. As a consequence, no extreme point of any face $c_{k} \boldsymbol{\Delta}_{\boldsymbol{J}^{(k)}}^{(k)}$ will become an inner point in

$$
\widehat{\boldsymbol{\Pi}}=\bigvee_{k \in \boldsymbol{K}_{0}} c_{k} \boldsymbol{\Pi}_{\boldsymbol{J}^{(k)}}^{(k)}
$$

which implies that $\operatorname{dim} \widehat{\Pi}=n-1$. Moreover the function

$$
x \mapsto\left\langle\mathfrak{n}_{F}, x\right\rangle
$$

is constant equal to 1 on $\widehat{\boldsymbol{\Pi}}$ and therefore $\mathfrak{n}_{F} \in \mathbb{R}^{n}$ is an outer normal (to the level 1) of $\widehat{\boldsymbol{\Pi}}$ which proves one direction of the theorem.

For the converse direction let us assume that there exists a set $\boldsymbol{K}_{0} \subset \boldsymbol{K}$ and faces

$$
\boldsymbol{\Delta}_{\boldsymbol{J}^{(k)}}^{(k)} \subset \boldsymbol{\Pi}^{(k)}
$$

of the corresponding prisms as well as positive numbers $c_{k} \in \mathbb{R}, k \in \boldsymbol{K}_{0}$ with the following properties:

1. For every $k \in \boldsymbol{K}_{0}$ the linear function $x \mapsto\left\langle n_{F}, \boldsymbol{x}\right\rangle$ is constant equal to 1 on the face $c_{k} \boldsymbol{\Delta}_{\boldsymbol{J}^{(k)}}^{(k)}$.

2. If two different faces $c_{k} \boldsymbol{\Delta}_{\boldsymbol{J}^{(k)}}^{(k)}$ and $c_{l} \boldsymbol{\Delta}_{\boldsymbol{J}^{(l)}}^{(l)}$ have extreme points on the same coordinate axis, then they coincide. 
3. The prism

$$
\widehat{\boldsymbol{\Pi}}=\bigvee_{k \in \boldsymbol{K}_{0}} c_{k} \boldsymbol{\Pi}_{\boldsymbol{J}^{(k)}}^{(k)}
$$

is $n$-dimensional and its outer normal (to the level 1 ) coincides with the outer normal $\mathfrak{n}_{F}$ of $F$.

Then let us consider the outer normal (to the level 1) $\mathfrak{n}_{S}$ of the n-dimensional prism $\widehat{\boldsymbol{\Pi}}$ as given by (3.1) The functional $f(\cdot)=\left\langle\mathfrak{n}_{S}, \cdot\right\rangle$ achieves its maximal values on the prisms

$$
\left\{\boldsymbol{\Pi}^{(k)}\right\}_{k \in \boldsymbol{K}_{0}}
$$

on the faces

$$
\boldsymbol{\Delta}_{\boldsymbol{J}^{(k)}}^{(k)} \subset \boldsymbol{\Pi}^{(k)}\left(k \in \boldsymbol{K}_{0}\right) .
$$

Since every maximal face of $\boldsymbol{\Pi}$ is the sum of faces of the prisms $\boldsymbol{\Pi}^{(k)}(k \in \boldsymbol{K})$, it follows that a translate of the set

$$
\sum_{k \in \boldsymbol{K}_{0}} c_{k} D_{\boldsymbol{J}^{(k)}}^{(k)}
$$

is contained in a maximal face $F \subset \boldsymbol{\Pi}$. Now we will prove that

$$
\operatorname{dim}\left(\sum_{k \in \boldsymbol{K}_{0}} c_{k} \boldsymbol{\Delta}_{\boldsymbol{J}^{(k)}}^{(k)}\right)=n-1
$$

holds true. Indeed, by condition 3) of the assertion the prism $\bigvee_{k \in \boldsymbol{K}_{0}} c_{k} \boldsymbol{\Pi}_{\boldsymbol{J}^{(k)}}^{(k)}$ is full-dimensional. Hence the Pareto-face has the dimension $(n-1)$. Since the Paretoface of $\widehat{\boldsymbol{\Pi}}$ is the convex hull of the faces $c_{k} \boldsymbol{\Delta}_{\boldsymbol{J}^{(k)}}^{(k)}\left(k \in \boldsymbol{K}_{0}\right)$, there exist edges of this face which are orthogonal to the vector $\mathfrak{n}_{S}$ and span a linear space of dimension $(n-1)$, which means that $\operatorname{dim} \sum_{k \in \boldsymbol{K}_{0}} c_{k} \boldsymbol{\Delta}_{\boldsymbol{J}^{(k)}}^{(k)}=n-1$.

Consequently, $F$ and the set $\sum_{k \in \boldsymbol{K}_{0}} c_{k} \boldsymbol{\Delta}_{\boldsymbol{J}^{(k)}}^{(k)}$ have the same outer normal. Since the set $\sum_{k \in \boldsymbol{K}_{0}} c_{k} \boldsymbol{\Delta}_{\boldsymbol{J}^{(k)}}^{(k)}$ and the prism $\widehat{\boldsymbol{\Pi}}$ have common outer normals, the converse direction is proved.

q.e.d.

Theorem 3.1 gives a possibility for an abstract description of the structure of faces in higher dimensions.

\section{Proposition 3.2.}

Let $\boldsymbol{a}=\left(a_{1}, \ldots, a_{n}\right)>\mathbf{0} \in \mathbb{R}^{n}$ and $\boldsymbol{b}=\left(b_{1}, \ldots, b_{n}\right)>\mathbf{0} \in \mathbb{R}^{n}$ be positive vectors and let $\Pi^{a}$ and $\Pi^{b}$ be the associated prisms.

Assume that the linear function $x \mapsto\langle a, x\rangle$ achieves its maximum over $\Pi^{b}$ in the extreme point $\boldsymbol{b}^{i_{0}}=b_{i_{0}} \boldsymbol{e}^{i_{0}} \in \boldsymbol{\Pi}^{\boldsymbol{b}}$, then

$$
\frac{b_{i_{0}}}{a_{i_{0}}} \mathbf{\Pi}^{a} \supseteq \boldsymbol{\Pi}^{b}
$$

and $b_{i_{0}} e^{i_{0}}$ is a common extreme point of both the prisms $\frac{b_{i_{0}}}{a_{i_{0}}} \Pi^{a}$ and $\boldsymbol{\Pi}^{b}$. 
Proof: Since the linear function $x \mapsto\langle a, x\rangle$ achieves its maximum over $\boldsymbol{\Pi}^{\boldsymbol{b}}$ in the extreme point $b_{i_{0}} \boldsymbol{e}^{i_{0}} \in \boldsymbol{\Pi}^{\boldsymbol{b}}$, we have

$$
\max \left\{\langle a, x\rangle \mid x \in \boldsymbol{\Pi}^{b}\right\}=\frac{b_{i_{0}}}{a_{i_{0}}}
$$

which implies

$$
\frac{b_{i_{0}}}{a_{i_{0}}} \boldsymbol{\Pi}^{a} \supseteq \Pi^{b}
$$

and that $\frac{b_{i_{0}}}{a_{i_{0}}} \boldsymbol{\Pi}^{\boldsymbol{a}}$ and $\boldsymbol{\Pi}^{b}$ have $b_{i_{0}} e^{i_{0}}$ as a common extreme point.

q.e.d.

\section{Faces and Permutations}

Tentatively we consider the case of two prisms. For convenience we use a simplified notation. For two vectors $\boldsymbol{a}=\left(a_{1}, \ldots, a_{n}\right)>\mathbf{0} \in \mathbb{R}^{n}$ and $\boldsymbol{b}=\left(b_{1}, \ldots, b_{n}\right)>\mathbf{0} \in \mathbb{R}^{n}$ we write

$$
\boldsymbol{A}=\boldsymbol{\Pi}^{\boldsymbol{a}}=\operatorname{conv}\left(\left\{\mathbf{0}, \boldsymbol{a}^{1}, \ldots, \boldsymbol{a}^{n}\right\}\right)
$$

and

$$
\boldsymbol{B}=\boldsymbol{\Pi}^{\boldsymbol{b}}=\operatorname{conv}\left(\left\{\boldsymbol{0}, \boldsymbol{b}^{1}, \ldots, \boldsymbol{b}^{n}\right\}\right)
$$

for the associated prisms. In this case, if the two prisms $\boldsymbol{A}$ and $\boldsymbol{B}$ have a common extreme point on their Pareto-faces then the following statement holds:

\section{Lemma 4.1.}

Suppose that $\boldsymbol{a}^{i}=\boldsymbol{b}^{i}$ holds true for some $i \in \boldsymbol{I}$.

Then for every index $l \neq i$ holds:

1. $\boldsymbol{a}^{l} \in \boldsymbol{A}$ is an extreme point of $\boldsymbol{A} \vee \boldsymbol{B}$ if and only if $\frac{a_{l}}{b_{l}} \geq \frac{a_{i}}{b_{i}}$

2. $\boldsymbol{b}^{l} \in \boldsymbol{B}$ is an extreme point of $\boldsymbol{A} \vee \boldsymbol{B}$ if and only if $\frac{a_{l}}{b_{l}} \leq \frac{a_{i}}{b_{i}}$.

Proof: In view of

$$
\boldsymbol{A}=\operatorname{conv}\left(\left\{\mathbf{0}, \boldsymbol{a}^{1}, \ldots, \boldsymbol{a}^{n}\right\}\right) \quad \text { and } \quad \boldsymbol{B}=\operatorname{conv}\left(\left\{\mathbf{0}, \boldsymbol{b}^{1}, \ldots, \boldsymbol{b}^{n}\right\}\right)
$$

we have

$$
\begin{gathered}
\boldsymbol{A} \vee \boldsymbol{B}=\operatorname{conv}\left(\left\{\boldsymbol{0}, \max \left\{a_{1}, b_{1}\right\} \boldsymbol{e}^{1}, \ldots, \max \left\{a_{i-1}, b_{i-1}\right\} \boldsymbol{e}^{i-1}, a_{k} \boldsymbol{e}^{i}, \max \left\{a_{i+1}, b_{i+1}\right\} \boldsymbol{e}^{i+1},\right.\right. \\
\left.\left.\ldots \ldots, \max \left\{a_{l}, b_{l}\right\} \boldsymbol{e}^{l}, \ldots, \max \left\{a_{n}, b_{n}\right\} \boldsymbol{e}^{n}\right\}\right)
\end{gathered}
$$

Now $\boldsymbol{b}^{l} \in \boldsymbol{B}$ is an extreme point of $\boldsymbol{A} \vee \boldsymbol{B}$ if and only if $a_{l} \leq b_{l}$ which is equivalent to $\frac{a_{l}}{b_{l}} \leq 1=\frac{a_{i}}{b_{i}}$, because $a_{i}=b_{i}$ by assumption.

The proof of the first statement is identical to this one.

q.e.d.

Now we consider the case of three prisms. We augment the notation by introducing

$$
\boldsymbol{C}=\boldsymbol{\Pi}^{c}=\operatorname{conv}\left(\left\{\mathbf{0}, \boldsymbol{c}^{1}, \ldots, \boldsymbol{c}^{n}\right\}\right)
$$




\section{Lemma 4.2.}

Let $\boldsymbol{a}, \boldsymbol{b}, \boldsymbol{c}$ be positive vectors and let $\boldsymbol{A}, \boldsymbol{B}, \boldsymbol{C}$ be the associated prisms. Assume that for an index $i \in \boldsymbol{I}$ the equations $\boldsymbol{a}^{i}=\boldsymbol{b}^{i}=\boldsymbol{c}^{i}$ hold true.

Then, for every $l \neq i$, the extreme point $\boldsymbol{c}^{l} \in \boldsymbol{C}$ is an extreme point of $\boldsymbol{A} \vee \boldsymbol{B} \vee \boldsymbol{C}$ as well if and only if

$$
\frac{a_{i}}{c_{i}} \geq \frac{a_{l}}{c_{l}} \quad \text { and } \quad \frac{b_{i}}{c_{i}} \geq \frac{b_{l}}{c_{l}}
$$

is satisfied.

Proof: The proof is identical to the proof of the previous Lemma 4.1. If $\boldsymbol{A}, \boldsymbol{B}$ and $\boldsymbol{C}$ are as above, then we have

$$
A \vee B \vee C=
$$

$\operatorname{conv}\left(\left\{\mathbf{0}, \max \left\{a_{1}, b_{1}, c_{1}\right\} \boldsymbol{e}^{1}, \ldots, \max \left\{a_{i-1}, b_{i-1}, c_{i-1}\right\} \boldsymbol{e}^{i-1}, a_{i} \boldsymbol{e}^{i}, \max \left\{a_{i+1}, b_{i+1}, c_{i+1}\right\} \boldsymbol{e}^{i+1}\right.\right.$,

$$
\left.\left.\ldots \ldots \ldots, \max \left\{a_{l}, b_{l}, c_{l}\right\} \boldsymbol{e}^{l}, \ldots, \max \left\{a_{n}, b_{n}, c_{n}\right\} \boldsymbol{e}^{n}\right\}\right) .
$$

Now $\boldsymbol{c}^{l} \in \boldsymbol{C}$ is an extreme point of $\boldsymbol{A} \vee \boldsymbol{B} \vee \boldsymbol{C}$ if and only if $a_{l} \leq c_{l}$ and $b_{l} \leq c_{l}$ which is equivalent to $\frac{a_{l}}{c_{l}} \leq 1=\frac{a_{i}}{c_{i}}$ and $\frac{b_{l}}{b_{l}} \leq 1=\frac{b_{i}}{c_{i}}$ because $a_{i}=b_{i}=c_{i}$ holds by assumption.

q.e.d.

\section{Proposition 4.3.}

Let $\boldsymbol{a}, \boldsymbol{b}$ be positive vectors and let $\boldsymbol{A}, \boldsymbol{B}$ be the associated prisms. For $i \in \boldsymbol{I}$ let

$$
\boldsymbol{F}_{\boldsymbol{A}}^{i}=\operatorname{conv}\left(\left\{\boldsymbol{a}^{l} \mid l \in\{1, \ldots, n\} \text { with } \frac{a_{l}}{b_{l}} \geq \frac{a_{i}}{b_{i}}\right\}\right)
$$

and

$$
\boldsymbol{F}_{\boldsymbol{B}}^{i}=\operatorname{conv}\left(\left\{\boldsymbol{b}^{l} \mid l \in\{1, \ldots, n\} \text { with } \frac{a_{l}}{b_{l}} \leq \frac{a_{i}}{b_{i}}\right\}\right) .
$$

Then, for every index $i \in \boldsymbol{I}$ the sum

$$
\boldsymbol{F}_{\boldsymbol{A}}^{i}+\boldsymbol{F}_{\boldsymbol{B}}^{i}
$$

is contained in a maximal face of $\boldsymbol{A}+\boldsymbol{B}$.

Proof: As $b_{i} \boldsymbol{A}$ and $a_{i} \boldsymbol{B}$ have a common extreme point, the result follows immediately from Lemma 4.1 and Theorem 3.1.

q.e.d.

We may now reformulate Proposition 4.3 in terms of permutations as follows.

Definition 4.4. Let $\boldsymbol{a}>\mathbf{0}, \boldsymbol{b}>\mathbf{0}$ be vectors of $\mathbb{R}^{n}$. A permutation $\pi: \boldsymbol{I} \longrightarrow \boldsymbol{I}$ is called a positioning permutation if the sequence of quotients

$$
\frac{a_{\pi_{(1)}}}{b_{\pi_{(1)}}} \geq \frac{a_{\pi_{(2)}}}{b_{\pi_{(2)}}} \geq \ldots \geq \ldots \geq \frac{a_{\pi_{(l)}}}{b_{\pi_{(l)}}} \geq \ldots \ldots \geq \frac{a_{\pi_{(n)}}}{b_{\pi_{(n)}}}
$$

is monotonically decreasing. We say that the vectors $\boldsymbol{a}, \boldsymbol{b}$ as well as the two prisms $\boldsymbol{A}=\boldsymbol{\Pi}^{\boldsymbol{a}}$ and $\boldsymbol{B}=\boldsymbol{\Pi}^{\boldsymbol{b}}$ are in regular position if their positioning permutation is uniquely defined. The positioning permutation is then denoted by $\pi_{(A, B)}$. 
A positioning permutation reflects the "relative position" of the associated prisms $\boldsymbol{A}$ and $\boldsymbol{B}$. Such a permutation does always exist, though it may not be uniquely determined. To avoid degenerated cases, we will henceforth always assume that all prisms are pairwise in regular position.

Corollary 4.5 (Faces and Permutations). Let $\boldsymbol{a}=\left(a_{1}, \ldots, a_{n}\right)>\mathbf{0} \in \mathbb{R}^{n}$ and $\boldsymbol{b}=\left(b_{1}, \ldots, b_{n}\right)>\mathbf{0} \in \mathbb{R}^{n}$ be given. Let $\pi_{(A, B)}$ be the positioning permutation of $\boldsymbol{A}$ and $\boldsymbol{B}$. Then, for every $i \in \boldsymbol{I}$ and

$$
\boldsymbol{F}_{\boldsymbol{A}}^{i}=\operatorname{conv}\left(\left\{\boldsymbol{a}^{l} \mid l \in\{1, \ldots, n\} \text { with } \pi_{(A, B)}^{-1}(l) \leq \pi_{(A, B)}^{-1}(i)\right\}\right)
$$

and

$$
\boldsymbol{F}_{\boldsymbol{B}}^{i}=\operatorname{conv}\left(\left\{\boldsymbol{b}^{l} \mid l \in\{1, \ldots, n\} \text { with } \pi_{(A, B)}^{-1}(l) \geq \pi_{(A, B)}^{-1}(i)\right\}\right)
$$

the sum

$$
\boldsymbol{F}_{\boldsymbol{A}}^{i}+\boldsymbol{F}_{\boldsymbol{B}}^{i}
$$

is contained in a maximal face of $\boldsymbol{A}+\boldsymbol{B}$.

Given two prisms $\boldsymbol{A}$ and $\boldsymbol{B}$, which are in regular position. Then the n-tuple

$$
\Pi\left(\frac{A}{B}\right)=\left[\pi_{(A, B)}(1), \pi_{(A, B)}(2), \ldots, \pi_{(A, B)}(n)\right] .
$$

is a strictly decreasing set of indices, the order induced by $\pi_{(A, B)}$, i.e.

$$
i \geq j \quad \Longleftrightarrow \quad \pi_{(A, B)}^{-1}(i) \leq \pi_{(A, B)}^{-1}(j) .
$$

Let us now introduce the abbreviation

$$
\boldsymbol{A B}(i)=\boldsymbol{F}_{\boldsymbol{A}}^{i}+\boldsymbol{F}_{\boldsymbol{B}}^{i},
$$

for the sum of two faces as defined above. Then for every $i \in \boldsymbol{I}$ the part of the face $\boldsymbol{A} \boldsymbol{B}(i)$ which belongs to $\boldsymbol{A}$ is determined by the indices which are greater or equal to $k$ in the order induced by $\pi_{(A, B)}$ and the part of the face $\boldsymbol{A} \boldsymbol{B}(i)$ which belongs to $\boldsymbol{B}$ is determined by the indices which are less or equal to $k$ in the order induced by $\pi_{(A, B)}$.

In terms of the positioning permutation we deduce from Lemma 4.2:

\section{Proposition 4.6.}

Suppose we are given 3 vectors $\boldsymbol{a}>\mathbf{0} \in \mathbb{R}^{n}, \quad \boldsymbol{b}>\mathbf{0} \in \mathbb{R}^{n}$, and $\boldsymbol{c}>\mathbf{0} \in \mathbb{R}^{n}$ and let $\boldsymbol{A}, \boldsymbol{B}$ and $\boldsymbol{C}$ be the associated prisms. Moreover, let $\pi_{(A, B)}$ and $\pi_{(A, C)}$ be the corresponding positioning permutations.

Then for every index $i \in \boldsymbol{I}$ the sum

$$
\boldsymbol{A B C}(i)=\boldsymbol{F}_{\boldsymbol{A}}^{i}+\boldsymbol{F}_{\boldsymbol{B}}^{i}+\boldsymbol{F}_{\boldsymbol{C}}^{i}
$$

with

$$
\boldsymbol{F}_{\boldsymbol{A}}^{i}=\operatorname{conv}\left(\left\{\boldsymbol{a}^{l} \mid l \in \boldsymbol{I} \text { with } \pi_{(A, B)}^{-1}(l) \leq \pi_{(A, B)}^{-1}(i) \text { and } \pi_{(A, C)}^{-1}(l) \leq \pi_{(A, C)}^{-1}(i)\right\}\right)
$$


$\boldsymbol{F}_{\boldsymbol{B}}^{i}=\operatorname{conv}\left(\left\{\boldsymbol{a}^{l} \mid l \in\{1, \ldots, n\} \quad\right.\right.$ with $\quad \pi_{(B, A)}^{-1}(l) \leq \pi_{(B, A)}^{-1}(i)$ and $\left.\left.\pi_{(B, C)}^{-1}(l) \leq \pi_{(B, C)}^{-1}(i)\right\}\right)$ and

$\boldsymbol{F}_{\boldsymbol{C}}^{i}=\operatorname{conv}\left(\left\{\boldsymbol{a}^{l} \mid l \in\{1, \ldots, n\} \quad\right.\right.$ with $\pi_{(C, A)}^{-1}(l) \leq \pi_{(C, A)}^{-1}(i)$ and $\left.\left.\pi_{(C, B)}^{-1}(l) \leq \pi_{(C, B)}^{-1}(i)\right\}\right)$ is contained in a maximal face of $\boldsymbol{A}+\boldsymbol{B}+\boldsymbol{C}$.

Proof: We know that $b_{i} c_{i} \boldsymbol{A}, a_{i} c_{i} \boldsymbol{B}$ and $a_{i} b_{i} \boldsymbol{C}$ have a common extreme point. Now the result follows immediately from Lemma 4.2 and Theorem 3.1.

q.e.d.

Note the the a similar condition stated in Proposition 4.6 is true for any finite sum.

\section{Proposition 4.7.}

Suppose we have 3 vectors $\boldsymbol{a}>\mathbf{0} \in \mathbb{R}^{n}, \quad \boldsymbol{b}>\mathbf{0} \in \mathbb{R}^{n}$, and $\boldsymbol{c}>\mathbf{0} \in \mathbb{R}^{n}$ and let $\boldsymbol{A}=\boldsymbol{\Pi}^{a}, \boldsymbol{B}=\boldsymbol{\Pi}^{b}$, and $\boldsymbol{C}=\boldsymbol{\Pi}^{c}$ be the associated prisms. Moreover, let $\pi_{(A, B)}$ and $\pi_{(A, C)}$ be the corresponding positioning permutations.

If for two indices $i, l \in \boldsymbol{I}$ we have:

- $i \geq l$ in the order induced by $\pi_{(A, B)}$,

- $l \geq i$ in the order induced by $\pi_{(A, C)}$,

then $\boldsymbol{A B}(l)$ and $\boldsymbol{A C}(i)$ have a parallel edge which is parallel to the line segment between $\boldsymbol{a}^{i}$ and $\boldsymbol{a}^{l}$ and conversely.

Proof: By Proposition 4.3 we have:

$$
\begin{gathered}
\boldsymbol{F}_{\boldsymbol{A}}^{l}=\operatorname{conv}\left(\left\{\boldsymbol{a}^{r} \mid r \in \boldsymbol{I} \text { with } r \geq l \text { in the order induced by } \pi_{(A, B)}\right\}\right), \\
\boldsymbol{F}_{\boldsymbol{B}}^{l}=\operatorname{conv}\left(\left\{\boldsymbol{b}^{s} \mid s \in \boldsymbol{I} \quad \text { with } s \leq l \text { in the order induced by } \pi_{(A, B)}\right\}\right), \\
\boldsymbol{F}_{\boldsymbol{A}}^{i}=\operatorname{conv}\left(\left\{\boldsymbol{a}^{s} \mid s \in \boldsymbol{I} \quad \text { with } s \geq i \text { in the order induced by } \pi_{(A, C)}\right\}\right)
\end{gathered}
$$

and

$$
\boldsymbol{F}_{\boldsymbol{C}}^{i}=\operatorname{conv}\left(\left\{\boldsymbol{c}^{s} \mid s \in \boldsymbol{I} \text { with } s \leq i \text { in the order induced by } \pi_{(A, C)}\right\}\right) .
$$

Since $i \geq l$ in the order induced by $\pi_{(A, B)}$ is follows, that the line segment $\left[\boldsymbol{a}^{i}, \boldsymbol{a}^{l}\right] \subset$ $\boldsymbol{F}_{\boldsymbol{A}}^{l}$. Analogously, since $l \geq i$ in the order induced by $\pi_{(A, B)}$ it follows, that the line segment $\left[\boldsymbol{a}^{i}, \boldsymbol{a}^{l}\right] \subset \boldsymbol{F}_{\boldsymbol{A}}^{i}$. Hence $\left[\boldsymbol{a}^{i}, \boldsymbol{a}^{l}\right] \subset \boldsymbol{A} \boldsymbol{B}(l)$ and $\left[\boldsymbol{a}^{i}, \boldsymbol{a}^{l}\right] \subset \boldsymbol{A} \boldsymbol{C}(i)$.

The converse direction is also clear.

q.e.d.

Proposition 4.7 gives a possibility to construct a maximal face by adjusting two parallel edges. We will use the notation:

$$
\boldsymbol{A B}(l) \boldsymbol{A C}(i)=\boldsymbol{F}_{\boldsymbol{A}}^{l}+\boldsymbol{F}_{\boldsymbol{B}}^{l}+\boldsymbol{F}_{\boldsymbol{C}}^{i}=\boldsymbol{F}_{\boldsymbol{A}}^{i}+\boldsymbol{F}_{\boldsymbol{B}}^{l}+\boldsymbol{F}_{\boldsymbol{C}}^{i}
$$

For the general case we have: 
Theorem 4.8.

Let $\boldsymbol{a}^{(1)}, \ldots, \boldsymbol{a}^{(K)} \in \mathbb{R}^{n}$ be a family of positive vectors and let

$$
\boldsymbol{\Pi}=\sum_{k=1}^{K} \boldsymbol{\Pi}^{(k)}
$$

with $\boldsymbol{\Pi}^{(k)}=\boldsymbol{\Pi}^{a^{(k)}}$ for $k \in \boldsymbol{K}$. Let $F \subset \boldsymbol{\Pi}$ be a maximal face with canonical reference system

$$
\mathfrak{J}_{F}=\left(\boldsymbol{K}_{0},\left[\boldsymbol{J}^{(k)}\right]_{k \in \boldsymbol{K}_{0}}\right)
$$

such that

$$
F=\sum_{k \in \boldsymbol{K}_{0}} \boldsymbol{\Delta}_{\boldsymbol{J}^{(k)}}^{(k)}+\boldsymbol{p}
$$

with a suitable sum $\boldsymbol{p}$ of extreme points of the remaining prisms is satisfied.

Then, for every two different indices $p, q \in \boldsymbol{K}_{0}$, we have for the index sets $\boldsymbol{J}^{(p)}$ and $\boldsymbol{J}^{(q)}$ that with respect to the order induced by $\pi_{\left(\boldsymbol{\Pi}^{(p)}, \boldsymbol{\Pi}^{(q)}\right)}$ the inequality

$$
\min \left\{\boldsymbol{J}^{(p)}\right\} \geq \max \left\{\boldsymbol{J}^{(q)}\right\}
$$

holds true.

Proof: This is an immediate consequence of Proposition 4.3.

q.e.d.

\section{The Adjustment Process: Two Examples}

Example 5.1. We consider 3 prisms in $\mathbb{R}^{4}$ which are given by:

$$
\begin{aligned}
& \boldsymbol{A}=\operatorname{conv}\{(0,0,0,0),(7,0,0,0),(0,5,0,0),(0,0,3,0),(0,0,0,1)\} \\
& \boldsymbol{B}=\operatorname{conv}\{(0,0,0,0),(2,0,0,0),(0,1,0,0),(0,0,10,0),(0,0,0,1)\} \\
& \boldsymbol{C}=\operatorname{conv}\{(0,0,0,0),(1,0,0,0),(0,6,0,0),(0,0,6,0),(0,0,0,5)\}
\end{aligned}
$$

For the permutations we have

$$
\begin{aligned}
& \pi_{(A, B)}=\left(\begin{array}{llll}
1 & 2 & 3 & 4 \\
2 & 1 & 4 & 3
\end{array}\right) \\
& \pi_{(A, C)}=\left(\begin{array}{lllll}
1 & 2 & 3 & 4 \\
1 & 2 & 3 & 4
\end{array}\right) \\
& \pi_{(B, C)}=\left(\begin{array}{lllll}
1 & 2 & 3 & 4 \\
1 & 3 & 2 & 4
\end{array}\right)
\end{aligned}
$$

Hence we have:

$$
\Pi\left(\frac{A}{B}\right)=[2,1,4,3]
$$




$$
\begin{aligned}
\Pi\left(\frac{A}{C}\right) & =[1,2,3,4] \\
\Pi\left(\frac{B}{C}\right) & =[1,3,2,4]
\end{aligned}
$$

Table of Faces

\begin{tabular}{lllll}
$\begin{array}{l}\text { Permu- } \\
\text { tation }\end{array}$ & $\begin{array}{l}\text { Adjusting } \\
k=1\end{array}$ & $\begin{array}{l}\text { Adjusting } \\
k=2\end{array}$ & $\begin{array}{l}\text { Adjusting } \\
k=3\end{array}$ & $\begin{array}{l}\text { Adjusting } \\
k=4\end{array}$ \\
\hline$\pi_{(A, B)}$ & $\underbrace{\left[a_{1}, a_{2}\right]+\left[b_{1}, b_{3}, b_{4}\right]}_{\boldsymbol{A} \boldsymbol{B}(1)}$ & $\begin{array}{l}\boldsymbol{A B}(2)=\boldsymbol{B} \\
\pi_{(A, C)}\end{array}$ & $\boldsymbol{A C}(1)=\boldsymbol{C} \boldsymbol{B}(3)=\boldsymbol{A}$ & $\underbrace{\left[a_{1}, a_{2}, a_{3}\right]+\left[b_{3}, b_{4}\right]}_{\boldsymbol{A} \boldsymbol{B}(4)}$ \\
$\pi_{(B, C)}$ & $\boldsymbol{B C}(1)=\boldsymbol{C}$ & $\underbrace{\left[b_{1}, a_{2}\right]+\left[c_{2}, c_{3}, c_{4}\right]}_{\boldsymbol{B C}(2)}$ & $\underbrace{\left[a_{1}(3)\right.}_{\boldsymbol{A}(2)}$ & $\boldsymbol{A} \boldsymbol{C}(4)=\boldsymbol{A}$
\end{tabular}

This describes 9 faces. The last face is constructed by Theorem 4.8, it is:

$$
F=\left[a_{1}, a_{2}\right]+\left[b_{1}, b_{3}\right]+\left[c_{2}, c_{4}\right],
$$

because we have:

- $\min \{1,2\} \geq \max \{1,3,4\}$ in the order induced by $\pi_{(A, B)}$,

- $\min \{1,2\} \geq \max \{2,3,4\}$ in the order induced by $\pi_{(A, C)}$,

- $\min \{1,3\} \geq \max \{2,3,4\}$ in the order induced by $\pi_{(B, C)}$.

Hence the index set which belongs to $A$ is $\{1,2\}$, the index set which belongs to $B$ is $\{1,3,4\} \cap\{1,3\}=\{1,3\}$, as follows from the inequalities implied by the orders of $\pi_{(A, B)}$ and $\pi_{(B, C)}$. Analogously the set of indices $\{2,4\}$ belongs to $C$, because $\{2,4\}=\Pi\left(\frac{B}{C}\right) \backslash\{1,3\}$.

The corresponding system of inequalities for $\boldsymbol{A}+\boldsymbol{B}+\boldsymbol{C}$ is:

$$
\begin{aligned}
& 30 x_{1}+30 x_{2}+5 x_{3}+6 x_{4} \leq 300 \\
& 5 x_{1}+7 x_{2}+x_{3}+10 x_{4} \leq 95 \\
& 5 x_{1}+10 x_{2}+x_{3}+2 x_{4} \leq 70 \\
& 5 x_{1}+10 x_{2}+x_{3}+5 x_{4} \leq 85 \\
& 150 x_{1}+210 x_{2}+35 x_{3}+42 x_{4} \leq 1610 \\
& 15 x_{1}+21 x_{2}+35 x_{3}+42 x_{4} \leq 665 \\
& 15 x_{1}+21 x_{2}+35 x_{3}+105 x_{4} \leq 980 \\
& 25 x_{1}+30 x_{2}+5 x_{3}+6 x_{4} \leq 255 \\
& 10 x_{1}+14 x_{2}+7 x_{3}+70 x_{4} \leq 490 \\
& 25 x_{1}+35 x_{2}+5 x_{3}+7 x_{4} \leq 260 .
\end{aligned}
$$


The polytope $\boldsymbol{A}+\boldsymbol{B}+\boldsymbol{C}$ has the following 21 extreme points:

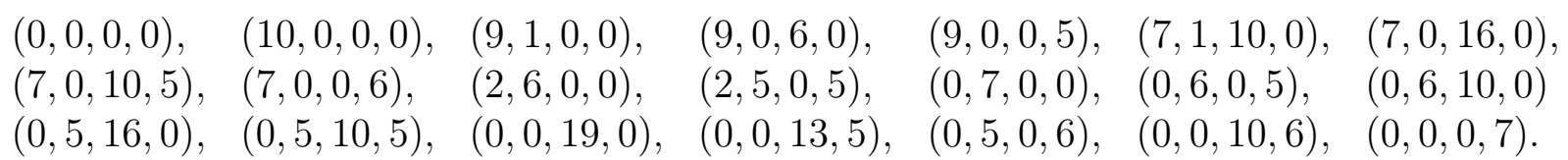

\section{Example 5.2.}

We consider 4 prisms in $\mathbb{R}^{5}$ which are given by:

$$
\begin{aligned}
& \boldsymbol{A}=\operatorname{conv}\{(0,0,0,0,0),(7,0,0,0,0),(0,5,0,0,0),(0,0,3,0,0),(0,0,0,1,0),(0,0,0,0,9)\} \\
& \boldsymbol{B}=\operatorname{conv}\{(0,0,0,0,0),(2,0,0,0,0),(0,1,0,0,0),(0,0,10,0,0),(0,0,0,1,0),(0,0,0,0,3)\} \\
& \boldsymbol{C}=\operatorname{conv}\{(0,0,0,0,0),(1,0,0,0,0),(0,1,0,0,0),(0,0,4,0,0),(0,0,0,5,0),(0,0,0,0,2)\} \\
& \boldsymbol{D}=\operatorname{conv}\{(0,0,0,0,0),(2,0,0,0,0),(0,3,0,0,0),(0,0,24,0,0),(0,0,0,4,0),(0,0,0,0,8)\}
\end{aligned}
$$

For these prisms we have:

$$
\begin{aligned}
& \Pi\left(\frac{A}{B}\right)=[2,1,5,4,3] \\
& \Pi\left(\frac{A}{C}\right)=[1,2,5,3,4] \\
& \Pi\left(\frac{A}{D}\right)=[1,2,5,4,3] \\
& \Pi\left(\frac{B}{C}\right)=[3,1,5,2,4] \\
& \Pi\left(\frac{B}{D}\right)=[1,3,5,2,4] \\
& \Pi\left(\frac{C}{D}\right)=[4,1,2,5,3]
\end{aligned}
$$

From Proposition 3.2 it follows that

$$
\begin{aligned}
& \boldsymbol{A B}(3)=\boldsymbol{A C}(4)=\boldsymbol{A D}(3)=\boldsymbol{A}, \\
& \boldsymbol{A B}(2)=\boldsymbol{B} \boldsymbol{C}(4)=\boldsymbol{B} \boldsymbol{D}(4)=\boldsymbol{B}, \\
& \boldsymbol{A C}(1)=\boldsymbol{B} \boldsymbol{C}(3)=\boldsymbol{C D}(3)=\boldsymbol{C}, \\
& \boldsymbol{A D}(1)=\boldsymbol{B D}(1)=\boldsymbol{C D}(4)=\boldsymbol{D} .
\end{aligned}
$$

Next we have 12 faces generated by two prisms, where one face of the generating prisms is one-dimensional. This are the faces: 


$$
\begin{array}{llllll}
\boldsymbol{A} \boldsymbol{B}(1)=\left[a_{1}, a_{2}\right]+\left[b_{1}, b_{3}, b_{4}, b_{5}\right] & , & \boldsymbol{A} \boldsymbol{B}(4)=\left[b_{3}, b_{4}\right]+\left[a_{1}, a_{2}, a_{3}, a_{5}\right] & \text { from } & \Pi\left(\frac{A}{B}\right) \\
\boldsymbol{A C}(2)=\left[a_{1}, a_{2}\right]+\left[c_{2}, c_{3}, c_{4}, c_{5}\right] & , & \boldsymbol{A} \boldsymbol{C}(3)=\left[c_{3}, c_{4}\right]+\left[a_{1}, a_{2}, a_{3}, a_{5}\right] & \text { from } & \Pi\left(\frac{A}{C}\right) \\
\boldsymbol{A} \boldsymbol{D}(2)=\left[a_{1}, a_{2}\right]+\left[d_{2}, d_{3}, d_{4}, d_{5}\right] & , & \boldsymbol{A} \boldsymbol{D}(4)=\left[d_{3}, d_{4}\right]+\left[a_{1}, a_{2}, a_{4}, a_{5}\right] & \text { from } & \Pi\left(\frac{A}{B}\right) \\
\boldsymbol{B C}(1)=\left[b_{1}, b_{3}\right]+\left[c_{1}, c_{2}, c_{4}, c_{5}\right] & , & \boldsymbol{B C}(2)=\left[c_{2}, c_{4}\right]+\left[b_{1}, b_{2}, b_{3}, b_{5}\right] & \text { from } & \Pi\left(\frac{A}{B}\right) \\
\boldsymbol{B D}(3)=\left[b_{1}, b_{3}\right]+\left[d_{2}, d_{3}, d_{4}, d_{5}\right] & , & \boldsymbol{B C}(2)=\left[d_{2}, d_{4}\right]+\left[b_{1}, b_{2}, b_{3}, b_{5}\right] & \text { from } & \Pi\left(\frac{A}{B}\right) \\
\boldsymbol{C D}(1)=\left[c_{1}, c_{4}\right]+\left[d_{1}, d_{2}, d_{3}, d_{5}\right] & , & \boldsymbol{C D}(5)=\left[d_{3}, d_{5}\right]+\left[c_{1}, c_{2}, c_{4}, c_{5}\right] & \text { from } & \Pi\left(\frac{A}{B}\right)
\end{array}
$$

There are 6 faces generated by two prisms, where both faces of the generating prisms is two-dimensional. This faces are generated by the middle elements in the ordered 5-tupels $\Pi\left(\frac{A}{B}\right)$, etc:

$$
\begin{array}{llll}
\boldsymbol{A B}(5) & =\left[a_{1}, a_{1} a_{5}\right]+\left[b_{3}, b_{4}, b_{5}\right] & \text { from } & \Pi\left(\frac{A}{B}\right) \\
\boldsymbol{A C}(5)=\left[a_{1}, a_{2}, a_{5}\right]+\left[c_{3}, c_{4}, c_{5}\right] & \text { from } & \Pi\left(\frac{A}{C}\right) \\
\boldsymbol{A D}(5)=\left[a_{1}, a_{2}, a_{5}\right]+\left[d_{3}, d_{4}, d_{5}\right] & \text { from } & \Pi\left(\frac{A}{B}\right) \\
\boldsymbol{B} \boldsymbol{C}(5)=\left[b_{1}, b_{3}, b_{5}\right]+\left[c_{2}, c_{4}, c_{5}\right] & \text { from } & \Pi\left(\frac{A}{B}\right) \\
\boldsymbol{B D}(5)=\left[b_{1}, b_{3}, b_{5}\right]+\left[d_{3}, d_{4}, d_{5}\right] & \text { from } & \Pi\left(\frac{A}{B}\right) \\
\boldsymbol{C D}(2)=\left[c_{1}, c_{2}, c_{4}\right]+\left[d_{2}, d_{3}, d_{5}\right] & \text { from } & \Pi\left(\frac{A}{B}\right)
\end{array}
$$

The next 12 faces are generated by three prisms. By Theorem 2.4 this faces can be generated by one or by two indices for the adjustment. We begin with the first 4 faces, which are generated by adjusting one index. This are the faces:

$$
\begin{aligned}
& \boldsymbol{A B C}(5)=\left[a_{1}, a_{2}, a_{5}\right]+\left[b_{3}, b_{5}\right]+\left[c_{4}, c_{5}\right] \text { for } \min \{1,2,5\}=\max \{3,4,5\} \text { in } \Pi\left(\frac{A}{B}\right), \\
& \min \{1,2,5\}=\max \{3,4,5\} \text { in } \Pi\left(\frac{A}{C}\right), \\
& \min \{1,3,5\}=\max \{2,4,5\} \text { in } \Pi\left(\frac{B}{C}\right), \\
& \boldsymbol{A} \boldsymbol{B} \boldsymbol{D}(5)=\left[a_{1}, a_{2}, a_{5}\right]+\left[b_{3}, b_{5}\right]+\left[d_{4}, d_{5}\right] \text { for } \min \{1,2,5\}=\max \{3,4,5\} \text { in } \Pi\left(\frac{A}{B}\right), \\
& \min \{1,2,5\}=\max \{3,4,5\} \text { in } \Pi\left(\frac{A}{D}\right), \\
& \min \{1,3,5\}=\max \{2,4,5\} \text { in } \Pi\left(\frac{B}{D}\right), \\
& \boldsymbol{A C D}(2)=\left[a_{1}, a_{2}\right]+\left[b_{2}, b_{4}\right]+\left[d_{2}, d_{3}, d_{5}\right] \text { for } \min \{1,2\}=\max \{2,3,4,5\} \text { in } \Pi\left(\frac{A}{B}\right), \\
& \min \{1,2\}=\max \{2,3,4,5\} \text { in } \Pi\left(\frac{A}{D}\right), \\
& \min \{1,2,4\}=\max \{2,3,5\} \text { in } \Pi\left(\frac{B}{D}\right) \text {, } \\
& \boldsymbol{A C D}(5)=\left[a_{1}, a_{2}, a_{5}\right]+\left[c_{4}, c_{5}\right]+\left[d_{3}, d_{5}\right] \text { for } \min \{1,2,5\}=\max \{3,4,5\} \text { in } \Pi\left(\frac{A}{C}\right), \\
& \min \{1,2,5\}=\max \{3,4,5\} \text { in } \Pi\left(\frac{A}{D}\right) \text {, } \\
& \min \{1,2,4,5\}=\max \{3,5\} \text { in } \Pi\left(\frac{C}{D}\right) \text {, }
\end{aligned}
$$


The next 8 faces are:

$$
\begin{aligned}
& (\boldsymbol{A B}(1))(\boldsymbol{A C}(2))=\left[a_{1}, a_{2}\right]+\left[b_{1}, b_{3}, b_{5}\right]+\left[c_{2}, c_{4}\right] \quad \text { for } \quad \min \{1,2\}=\max \{1,3,4,5\} \text { in } \Pi\left(\frac{A}{B}\right), \\
& \min \{1,2\}=\max \{2,3,4,5\} \text { in } \Pi\left(\frac{A}{C}\right), \\
& \text { and } \min \{1,3,5\}>\max \{2,4\} \text { in } \Pi\left(\frac{B}{C}\right) \text {, } \\
& \text { or } \min \{1,3\}>\max \{2,4,5\} \text { in } \Pi\left(\frac{B}{C}\right) \text {, } \\
& (\boldsymbol{A C}(2))(\boldsymbol{B C}(5))=\left[a_{1}, a_{2}\right]+\left[b_{3}, b_{5}\right]+\left[c_{2}, c_{4}, c_{5}\right] \quad \text { for } \min \{2,3,4,5\}=\max \{1,2\} \text { in } \Pi\left(\frac{C}{A}\right), \\
& \min \{2,4,5\}=\max \{1,3,5\} \text { in } \Pi\left(\frac{C}{B}\right) \text {, } \\
& \min \{1,2\}>\max \{3,4,5\} \text { in } \Pi\left(\frac{A}{B}\right) \text {, } \\
& (\boldsymbol{A B}(1))(\boldsymbol{B D}(5))=\left[a_{1}, a_{2}\right]+\left[b_{1}, b_{3}, b_{5}\right]+\left[c_{4}, c_{5}\right] \quad \text { for } \quad \min \{1,3,4,5\}=\max \{1,2\} \text { in } \Pi\left(\frac{B}{A}\right), \\
& \min \{1,3,5\}=\max \{2,4,5\} \text { in } \Pi\left(\frac{B}{D}\right) \text {, } \\
& \min \{1,2\}>\max \{3,4,5\} \text { in } \Pi\left(\frac{A}{D}\right) \text {, } \\
& (\boldsymbol{A B}(1))(\boldsymbol{B D}(3))=\left[a_{1}, a_{2}\right]+\left[b_{1}, b_{3}, b_{5}\right]+\left[c_{2}, c_{4}\right] \text { for } \\
& \text { and } \min \{1,2\}>\max \{3,4,5\} \text { in } \Pi\left(\frac{A}{D}\right) \text {, } \\
& \text { or } \min \{1,2,5\}>\max \{3,4\} \text { in } \Pi\left(\frac{A}{D}\right) \text {, } \\
& (\boldsymbol{A C}(2))(\boldsymbol{C D}(5))=\left[a_{1}, a_{2}\right]+\left[c_{2}, c_{4}, c_{5}\right]+\left[d_{3}, d_{5}\right] \text { for } \min \{2,3,4,5\}=\max \{1,2\} \text { in } \Pi\left(\frac{C}{A}\right), \\
& \min \{1,2,4,5\}=\max \{3,5\} \text { in } \Pi\left(\frac{C}{D}\right) \text {, } \\
& \min \{1,2\}>\max \{3,4,5\} \text { in } \Pi\left(\frac{A}{D}\right), \\
& (\boldsymbol{C D}(2))(\boldsymbol{B D}(5))=\left[b_{1}, b_{3}, b_{5}\right]+\left[c_{2}, c_{4}\right]+\left[d_{2}, d_{5}\right] \quad \text { for } \quad \min \{2,4,5\}=\max \{1,3,5\} \text { in } \Pi\left(\frac{D}{B}\right), \\
& \min \{2,3,5\}=\max \{1,2,4\} \text { in } \Pi\left(\frac{D}{C}\right) \text {, } \\
& \min \{1,3,5\}>\max \{2,4\} \text { in } \Pi\left(\frac{B}{C}\right) \text {, } \\
& (\boldsymbol{B D}(3))(\boldsymbol{C D}(5))=\left[b_{1}, b_{3}\right]+\left[c_{2}, c_{4}, c_{5}\right]+\left[d_{3}, d_{5}\right] \quad \text { for } \\
& \min \{2,3,4,5\}=\max \{1,3\} \text { in } \Pi\left(\frac{D}{B}\right), \\
& \min \{3,5\}=\max \{1,2,4,5\} \text { in } \Pi\left(\frac{D}{C}\right) \text {, } \\
& \min \{1,3\}>\max \{2,4,5\} \text { in } \Pi\left(\frac{B}{C}\right) \text {, } \\
& (\boldsymbol{B D}(3))(\boldsymbol{C D}(2))=\left[b_{1}, b_{3}\right]+\left[c_{2}, c_{4}\right]+\left[d_{2}, d_{3}, d_{4}\right] \quad \text { for } \min \{2,3,4,5\}=\max \{1,3\} \text { in } \Pi\left(\frac{D}{B}\right), \\
& \min \{2,3,5\}=\max \{1,2,4\} \text { in } \Pi\left(\frac{D}{C}\right), \\
& \text { and } \min \{1,2\}>\max \{2,4,5\} \text { in } \Pi\left(\frac{B}{C}\right) \text {, } \\
& \text { or } \min \{1,3,5\}>\max \{2,4\} \text { in } \Pi\left(\frac{B}{C}\right) \text {, }
\end{aligned}
$$

There is one face which is generated by all four prisms, i.e.

$$
(\boldsymbol{A B}(1))(\boldsymbol{A} C(2))\left(\boldsymbol{B} D(3)=\left[a_{1}, a_{2}\right]+\left[b_{1}, b_{3}\right]+\left[c_{2}, c_{4}\right]+\left[d_{3}, d_{5}\right] .\right.
$$

This face can be determined by Theorem 4.8 from the following inequalities: 


$\begin{array}{lll}2>1>3 & \text { in } & \Pi\left(\frac{A}{D}\right), \\ 1>2 & \text { in } & \Pi\left(\frac{A}{C}\right), \\ \min \{1,3\}>2 & \text { in } & \Pi\left(\frac{B}{C}\right), \\ 1>3 & \text { in } & \Pi\left(\frac{B}{D}\right), \\ 2>3 & \text { in } & \Pi\left(\frac{C}{D}\right) .\end{array}$

This examples show that it is possible to determine all faces of the Minkowski sum of prisms for small dimensions.

\section{References}

[1] Ewald, G. (1996): Combinatorial Convexity and Algebraic Geometry, Springer Verlag, Berlin, Heidelberg, New York.

[2] Pallaschke, D. and Urbański, R. (2002): Pairs of Compact Convex Sets Fractional Arithmetic with Convex Sets, Mathematics and its Applications, Vol. 548, Kluwer Acad. Publ. Dordrecht.

[3] Pallaschke, D. and Rosenmüller, J. (2004): Cephoids: Minkowski Sums of Prisms, Working Paper No. 360, Institute of Mathematical Economics, University of Bielefeld.

[4] Pallaschke, D. and Rosenmüller, J. (2004): A Superadditive Solution, Working Paper No. 361, Institute of Mathematical Economics, University of Bielefeld.

[5] Rosenmüller, J. (2000): Game Theory: Stochastics, information, strategies and Cooperation, Theory and Decision Library, Series C, Vol 25,Kluwer Acad. Publ. Dordrecht.

[6] Schneider, R. (1993): Convex Bodies: The Brunn-Minkowski Theory, Encyclopedia of Mathematics and its Applications, Vol.44, Cambridge University Press.

[7] Weil, W. (1980): Eine Charakterisierung von Summanden konvexer Körper, Archiv der Mathematik 34, 283-288. 